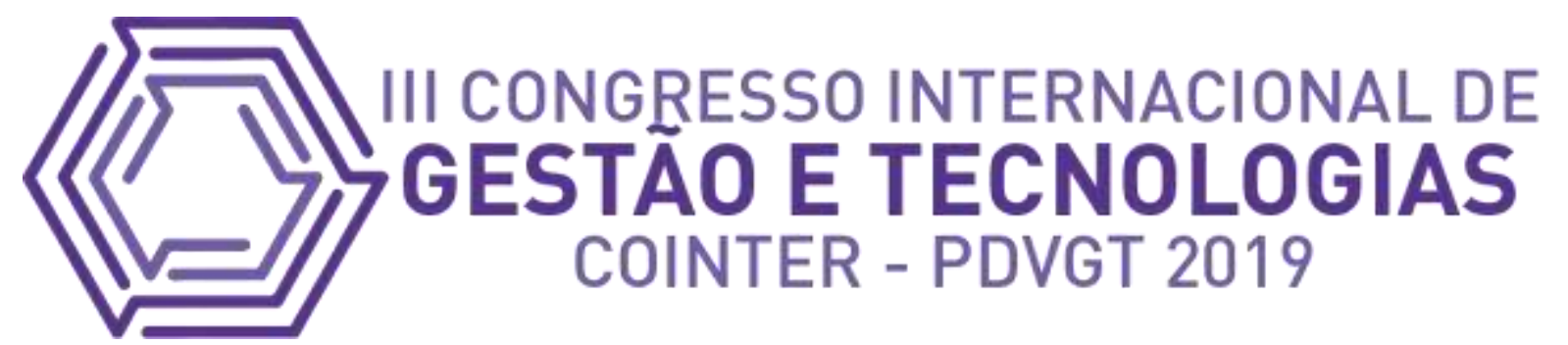

\title{
REDES DE COOPERAÇÃO E ATIVIDADES DE EXTENSÃO: ESTUDO EM UM CURSO DE LICENCIATURA.
}

\section{REDES DE COOPERACIÓN Y ACTIVIDADES DE EXTENSIÓN: UM ESTUDIO EN UM CURSO DE LICENCIA}

\section{COOPERATION NETWORKS AND EXTENSION ACTIVITIES: A STUDY IN A LICENSE COURSE}

\author{
Apresentação: Comunicação Oral
}

Kaline Soares da Silva ${ }^{1}$; Maria José Cavalcante ${ }^{2}$; Elielma Josefa de Moura ${ }^{3}$; Erick Viana da Silva $^{4}$

\section{DOI: https://doi.org/10.31692/2358-9728.VICOINTERPDVG.2019.0004}

\section{Resumo}

A Instituição de Ensino Superior estudada desde sua criação tem o desafio de consolidar o escopo de sua criação, desenvolvendo suas atividades baseando-se neste escopo, sendo esse Ensino, Pesquisa e Extensão, que esses três sejam indissociáveis. A extensão é um trabalho desenvolvido a partir da problemática encontrada na sociedade, visto isso a academia por sua vez tem como função desenvolver trabalhos com a intensão de amenizar ou resolver estes, dando para a sociedade a resposta de todo o trabalho desenvolvido, por meio de redes de cooperação e da gestão do conhecimento esse plano de trabalho objetivou a identificação de trabalhos interinstitucionais desenvolvidos na área de extensão no curso de Licenciatura em Química de uma Instituição de Ensino Superior, para isso foram estudados os editais de extensão publicados por esta e com os resultados desses editais foi feita uma análise do crescimento da quantidade de extensionistas, também foi feita uma análise de alguns documentos, como o Plano Nacional de Extensão, para se obter os dados sobre a situação atual da extensão universitária no País e como ela se comporta dentro das instituições. Foi possível concluir que a extensão dentro do campus tem sido desenvolvida com o incentivo de

\footnotetext{
${ }^{1}$ Engenharia Agrícola, INACAP, kalis.soares8@gmail.com

${ }^{2}$ Bacharelado em Agronomia, IFPE, janecavalcante@gmail.com

${ }^{3}$ Bacharelado em Agronomia, IFPE, elielmamoura@outlook.com

${ }^{4}$ Mestre em Administração, IFPE, erick.viana@recife.ifpe.edu.br
} 
bolsas, o aumento de projetos cadastrados e estes trabalhos estão sendo desenvolvidos para estudantes do Ensino Médio, com a intensão de facilitar o aprendizado destes na disciplina de química, sendo assim criada uma rede de cooperação entre o campus e a escola além do conhecimento que antes era apenas do extensionista sendo passado para o a comunidade, tornando esse conhecimento explícito.

Palavras-Chave: Extensão, Gestão do Conhecimento, Redes de Cooperação

\section{Resumen}

La Institución de Educación Superior estudiada desde su inicio tiene el desafio de consolidar el alcance de su creación, desarrollando sus actividades en función de este alcance, siendo que la Enseñanza, la Investigación e la Extensión, estos tres son inseparables. La extensión es un trabajo desarollado a partir de la problemática encontrada em la sociedade, ya que la academia a su vez tiene la función de desarrollar trabajos com la intención de suavizarlos o resolverlos, dando a la sociedad la respuesta de todo el trabajo desarrollado, a través de Redes de cooperación y gestión del conocimiento. Este trabajo tuvo como objetivo identificar trabajos interinstitucionales desarrollados em el área de extensión em el Grado em Química de uma Institución de Educación Superior, para tal fin los edictos de extensión publicados por este y com el. Los resultados de estos edictos se hicieron um análisis del crecimiento del número de extensionistas, también um análisis de algunos documentos, como el Plan Nacional de Extensión, para obtener datos sobre la situación actual de la extensión universitária em el país y cómo se comporta de las instituciones. Se concluyó que la extensión em el campus se há desarrollado com el incentivo de becas, el aumento de proyectos registrados y estos trabajos se están desarrollando para estudiantes de secundaria, com la intención de facilitar su aprendizaje em la disciplina de quimica. Se há creado una red de cooperación entre el campus y la escuela y el conocimiento que antes era solo el del extensionista se transmitia a la comunidade, lo que hace que este conocimiento sea explicito.

Palabras Clave: Extensión, Gestión del Conocimiento, Redes de cooperacion 


\begin{abstract}
The Higher Education Institution studied since its inception has the challenge of consolidating the scope of its creation, developing its activities based on this scope, being that Teaching, Research and Extension, these three are inseparable. The extension is a work developed from the problematic found in society, since the academy in turn has the function to develop works with the intention of softening or solving these, giving to society the answer of all the work developed, through Cooperation networks and knowledge management. This work plan aimed to identify interinstitutional works developed in the extension area in the Degree in Chemistry of a Higher Education Institution, for this purpose the extension edicts published by this and with the. The results of these edicts were made an analysis of the growth of the number of extensionists, also an analysis of some documents, such as the National Extension Plan, to obtain data on the current situation of the university extension in the country and how it behaves in. of the institutions. It was concluded that the extension on campus has been developed with the incentive of scholarships, the increase of registered projects and these works are being developed for high school students, with the intention of facilitating their learning in the chemistry discipline. A network of campus-school co-operation is created, and knowledge that was once only the extensionist's passing on to the community, making that knowledge explicit.
\end{abstract}

Keywords: Extension, Knowledge Management, Cooperation Networks

\title{
Introdução
}

Desde 2008 quando da criação da Instituição de Ensino Superior, tem nos últimos anos cumprido o desafio de consolidar os objetivos de desenvolver políticas de Ensino, Pesquisa e Extensão na busca da integração e indissociabilidade destes pilares. Como justificativa da importância desse plano de atividades podemos citar a necessidade de percebermos de forma integrada e sistêmica a importância dos impactos que processos de cooperação interinstitucionais podem trazer à formação dos estudantes. Assim sendo, esse trabalho de pesquisa objetivou identificar as atividades de Extensão realizadas no curso de 
Licenciatura em Química que possuam relação com ações de formação de redes e cooperação, especificamente na cooperação interinstitucional.

\section{Fundamentação Teórica}

\section{REDES DE COOPERAÇÃO}

Segundo Olave et al. (2001), com o crescimento da internacionalização da economia intensificou-se a necessidade de reorganizar os fatores produtivos e os modos de gestão empresarial, com o intuito de compatibilizar a organização com padrões internacionais de qualidade e produtividade. Dentre as estratégias para esta reorganização, a mesma cita a formação de redes entre empresas, sendo essa uma prática atual que pretende garantir a sobrevivência e a competitividade entre as pequenas e médias empresas.

Segundo a análise organizacional a administração faz um mister para gerenciar seus processos de forma integrada e orientada para seus objetivos (SILVA et al, 2017). Uma das novas formas que vem se solidificando no processo da reestruturação industrial é a que se refere às formas de relações intra e interempresas (OLAVE et al, 2001).

As atividades de reestruturação, conduziram à uma nova formulação das estratégias das grandes empresas. Partindo dessa, as negociações entre os agentes econômicos ganham novos contornos e passam a integrar o rol dos condicionantes do aumento da competitividade industrial (YOGUEL et al., 1990).

Essa mudança no âmbito das relações industrial mundialmente, segundo De Souza (1993), é um movimento de transição que cabe evidenciar o intenso ritmo dos avanços tecnológicos, sendo esses dos equipamentos, técnicas e produtos, as crescentes flutuações do mercado, o aumento das incertezas na hora da tomada de decisão dos agentes econômicos e as empresas em muitos países defrontam-se com crescentes tensões nas áreas políticas, trabalhistas e de legislação.

Com todo esse cenário as redes de cooperação vêm tentar reduzir os custos de transação, sendo esses os custos que vão além da produção em si, aumentando assim a competitividade e a eficiência econômica.

O conceito de redes de cooperação surge como uma nova forma de organização do trabalho e relacionamento entre empresas. A cooperação entre organizações com o objetivo de 
obter soluções coletivas tem recebido crescente atenção nos estudos e práticas organizacionais nas últimas três décadas (NOHRIA \& ECCLES, 1992).

As redes de cooperação têm a capacidade de facilitar a realização de ações conjuntas e a transação de recursos para alcançar objetivos organizacionais. Podem ser definidas como o conjunto de transações repetidas e sustentadas por configurações relacionais e estruturais dotadas de fronteiras dinâmicas e elementos interconectados (TODEVA, 2006). Este novo modelo propõe maior competitividade à essas organizações, aliando a flexibilidade presente no sistema de redes (BALESTRIN,2008).

GRANDIORI\&SODA (1995), identifica três tipos básicos de redes: redes sociais, redes burocráticas e redes proprietárias. As redes sociais são aquelas onde os indivíduos não tem nenhum contrato formal, e ela pode ser subdividida em redes sociais simétricas onde não existe uma empresa com um poder diferenciadas sendo assim todas tem o mesmo poder e em redes sociais assimétricas, é quando existe a presença de um agente central. As redes burocráticas são aquelas que possuem um contrato formal e as redes proprietárias que são aquelas que na maioria das vezes quando relacionam o investidor de um lado e a empresa parceira de outro (OLAVE et al, 2001).

Segundo Oliver e Ebers (1998) e Caglio (1998), os estudos sobre redes interorganizacionais foram conduzidos a partir de algumas correntes teóricas. A primeira, a da Economia Industrial, é utilizada nas pesquisas sobre redes para permitir que se entenda como os diferentes ganhos econômicos de produção - economias de escala, de escopo e de especialização - explicam a eficiência das redes (BALESTRIN, 2010). Esta identificou diferentes classes de custos de produção, como economias de escala, de escopo, de especialização e de experiência, como variáveis explicativas da eficiência das redes (ECCLES, 1981;TURATI, 1990; TEECE, 1980).

A segunda, a das Estratégias Interorganizacionais, de acordo com as quais a configuração em rede é vista como um fator estratégico no alcance e na manutenção de vantagens competitivas (MARCON E MOINET, 2000; FAYARD, 2000; JARILLO, 1988).

A terceira corrente, a da Teoria da Dependência de Recursos, seu foco de interesse é entender o processo por meio do qual as organizações reduzem suas dependências ambientais, utilizando várias estratégias para aumentar seu próprio poder. Essa teoria distingue entre tipos de dependência de recursos, materiais ou imateriais, como possíveis determinantes na formação de redes (BALESTRIN, 2010). 
A quarta corrente a da abordagem das Redes Sociais (social networks) evidenciou que a posição dos atores em uma rede influencia a organização de seus membros e apresenta forte influência nas inter-relações junto à rede (POWELL ET AL, 1996; BURT, 1992). A quinta, a da Teoria Institucional, constatou que as organizações buscam ganhar legitimidade em seu ambiente institucional no momento de se estruturarem em rede (DIMAGGIO E POWELL, 1983; GRABHER, 1993). E por fim a sexta corrente representada pelas Teorias Críticas, que questionaram o argumento da eficiência na formação das redes e destacaram que as redes são formadas por representarem poderosos instrumentos na formação de elites e classes dominantes, bem como o exercício do poder e da dominação (PERUCCI E POTTER, 1989; SALANCIK, 1995).

Para Casarotto(1999), por conta da crescente complexidade das tarefas dentro das organizações é necessário parcerias entre as empresas para trabalhar de forma associada, o mesmo considera que é pouco possível que uma pequena empresa consiga desenvolver sozinha todas as etapas de uma produção, logo se há a formação de redes de cooperação as pequenas e médias empresas podem ser tornar igualmente competitivas ao agregarem vantagem, muitas vezes estas são mais rápidas e flexíveis que as grandes empresas.

Entre as mais recentes formações de redes entre empresas, estão os Clusters e as organizações virtuais. O conceito de cluster pode-se entender, como concentração setorial e geográfica de empresas. Dentre as características mais importantes temos o ganho de eficiência coletiva, entendida como a vantagem competitiva das economias externas locais e da ação conjunta (PORTER, 1998). Num cluster encontra-se o escopo para a divisão das tarefas entre as empresas, tanto como para especialização ou para inovação e para elementos essenciais para a competição além dos mercados da região (OLAVE et. al, 2001)

\section{GESTÃO DO CONHECIMENTO}

No universo das instituições de ensino com protagonismo e sucesso em suas experiências formativas já se tornou um ponto pacífico a importância da gestão do conhecimento (GC). Instituições públicas e privadas ao redor do mundo implementam grupos internos de aprendizagem organizacional, capital humano, capital intelectual, enfim há uma miscelânea de conceitos aplicados a gestão de organizações que possuem no seu cerne a semente da valorização do conhecimento. Senge (2000), Probst et al (1997), Takeuchi e 
Nonaka(1997) denotam em suas obras a importância que o conhecimento teve durante a história da humanidade e sua cada vez mais intensa 3 utilização por parte das organizações.

Nonaka e Takeuchi (1997) firmam a tese de que o conhecimento de grande valor para a organização possui as seguintes dimensões, a primeira que é tácito, pois é extremamente relacionado à ação, aos procedimentos, às rotinas, às ideias, aos valores e à quesitos emocionais; é dinâmico, pois é construído dentro de relações sociais entre indivíduos, grupos e organizações; e humanista, pois é essencialmente relacionado à ação humana. De acordo com os autores, essas características fazem com que o conhecimento dificilmente possa ser gerenciado.

Nessa mesma linha de pensamento os autores Nonaka Toyama e Conno (2002), afirmam que um fator estratégico é a criação de novos conhecimentos, pois é mais viável que gerencia-los. Segundo os mesmos autores, as condições favoráveis para a criação desse novo conhecimento em uma nova organização seria pelo método SECI- Socialização, Externalização, Combinação, Internalização.

Nonaka e Takeuchi (1997) definem de conversão de conhecimento o processo com que as ações criam conhecimento, sendo por meio dessa conversão que o conhecimento tácito e explícito é expandido. Existem quatro modos de conversão de conhecimento: a socialização que é a conversão de conhecimento tácito em conhecimento tácito; a externalização, conversão de conhecimento tácito em conhecimento explícito, a combinação, conversão de conhecimento explícito em conhecimento explícito, e a internalização, conversão de conhecimento explícito em conhecimento tácito.

Nonaka, Toyama e Konno (2002) apresentam quatro grupos de "espaços de interação", esses são os que sustentam um modo particular de conversão de conhecimento nas fases do processo SECI, são os espaços de socialização de conhecimento, espaços de externalização de conhecimento, espaços de sistematização de conhecimento e espaços de internalização de conhecimento. Esses espaços de socialização permitem que o conhecimento seja socializado entre os indivíduos participantes da organização, por meio da interação cara a cara, onde os mesmos partilham de emoções, sentimentos e experiências, já os espaços de externalização são situações onde os indivíduos dividem suas experiências por meio de conversas, convertendo elas em termos comuns, nos espaços de sistematização oferecem um contexto de combinação com o novo conhecimento com aquele já existente na organização e por fim nos espações de internalização é onde o conhecimento que já foi socializado, 
externalizado e sistematizado seja novamente interpretado e internalizado pelos indivíduos na formação de novos conceitos e nas práticas do trabalho (BALESTRIN, 2005).

\section{EXTENSÃO UNIVERSITÁRIA}

Das três dimensões constitutivas da universidade, Pesquisa, Ensino e Extensão, a Extensão foi a última a surgir, seja por isso, seja por sua natureza intrinsecamente interdisciplinar, seja pelo fato de se realizar, em grande medida, além das salas de aulas e dos laboratórios, seja pelo fato de estar voltada para o atendimento de demandas por conhecimento e informação de um público amplo, difuso e heterogêneo, por tudo isso, talvez, as atividades de Extensão não têm sido adequadamente compreendidas e assimiladas pelas universidades (PAULA, 2013).

Segundo Serrano (2013), pensar na universidade a partir de seus objetivos básicos destes como a formação profissional, a geração de novos conhecimentos e a divulgação desses conhecimentos é um processo complexo, é nesse contexto é que a Extensão universitária está inserida, está apresenta uma diversidade conceitual e prática que interfere no modo de pensar e fazer no interior da academia.

A Extensão universitária é uma forma de ligação que deve existir entre a universidade e a comunidade na qual ela está inserida, uma espécie de rede permanente entre a universidade e os diversos setores da sociedade (SILVA et. al, 2011). A indissociabilidade ensino-pesquisa-Extensão reafirma a Extensão como um processo acadêmico e lhe justifica o adjetivo universitária (CÔRREA, 2003).

Rocha (2001), tem como origem da Extensão as universidades europeias medievais, em especial a Universidade de Bolonha, ele também aponta que na Inglaterra no século XIX, haviam práticas de Extensão universitária dadas com a participação dos estudantes em campanhas de saúde, na utilização do teatro escolar entre outros serviços.

No Brasil, desde 1911, inicialmente em São Paulo, que as atividades de Extensão têm se dado em instituições de ensino superior no Brasil, reproduzindo aqui as vertentes típicas da tradição europeia de Extensão: “educação continuada e educação voltada para as classes populares; Extensão voltada para a prestação de serviços na área rural" (NOGUEIRA, 2005). A Extensão universitária no país está prevista desde a legislação de 1931 que, mediante o Decreto $\mathrm{n}^{\circ} 19.851$, de 11/4/1931, estabeleceu as bases do sistema universitário brasileiro. 
Segundo o Plano Nacional de Extensão, elaborado pelo Fórum de Pró-Reitores de Extensão das Universidades Públicas Brasileiras e pela Secretaria do Ensino Superior do Ministério da Educação e do Desporto, a Extensão universitária é o processo educativo, cultural e científico que articula o ensino e a pesquisa de forma indissociável e viabiliza a relação transformadora entre universidade e sociedade.

Na Extensão universitária, ocorre uma troca de conhecimentos em que a academia também aprende com a comunidade sobre sua cultura, crenças e valores. Assim, a universidade pode planejar e executar as atividades de Extensão respeitando e não violando a história cultural já existente na comunidade. A universidade, através da Extensão, influencia e também é influenciada pela comunidade, ou seja, há uma troca de valores entre a universidade e o meio (SILVA, 1997).

Por meio da Extensão, a universidade tem a oportunidade de levar à comunidade os conhecimentos de que é detentora, os novos conhecimentos produzidos pela pesquisa e normalmente divulgados com o ensino. É uma forma de a universidade socializar e democratizar o conhecimento, levando-o aos não universitários. Assim, o conhecimento não se traduz em privilégio apenas da minoria que é aprovada no vestibular, mas difundido pela comunidade, consoante os próprios interesses dessa mesma comunidade (SILVA, 1997).

A relação social de impacto entre universidade e os setores presentes na sociedade deve ser antes de qualquer coisa, transformadora, ou seja, um instrumento de mudança em busca de melhoria da qualidade de vida. A bilateralidade deve ser essencial na relação entre a universidade e a comunidade, a academia deve construir uma relação de mão dupla com troca de saberes popular e acadêmico (CORRÊA, 2003).

\section{Metodologia}

A presente pesquisa é de natureza exploratória-descritiva, de campo, quantiqualitativa. O campo de Pesquisa foi constituído por um campus do Instituto Federal de Pernambuco. Os sujeitos de pesquisa foram os estudantes do curso de licenciatura em química. Foram utilizados documentos como editais de Extensão publicados pela Instituição de Ensino e documentos relacionados á Extensão Universitária. A pesquisa teve dois momentos: análise documental, o Plano Nacional de Extensão e os Editais de Extensão publicados no período estudado nessa pesquisa (2015-2018). 


\section{Resultados e Discussão}

A Instituição de Ensino Superior define como Extensão um espaço em que os campis promovem a articulação entre o saber acadêmico e a realidade socioeconômica e cultural da região onde está inserido, ou seja, podemos afirmar que a Extensão é a prática ligante entre o ensino e a pesquisa dentro dos Institutos Federais. O Plano Nacional de Extensão - PNE apresenta o princípio básico para a formação do profissional cidadão, sendo imprescindível a sua interação com a Sociedade (BRASIL, 2001).

A pró-reitoria de Extensão da Instituição de Ensino tem como competência propor normas e políticas sobre as atividades desenvolvidas pelos extensionistas e fazer todo o papel de fiscalização, acompanhamento e divulgação das atividades que estão sendo desenvolvidas pelo Instituto.

Nos editais que são lançados pela Instituição para cadastro dos Projetos de Extensão que são entendidos como uma ação processual e contínua de caráter educativo, social, cultural ou tecnológico, com objetivos e prazo determinado. Para serem submetidos á análise o projeto precisa contemplar algumas das áreas estabelecidas pela Pró-Reitoria da Instituição, que foram elencadas de acordo com o Programa Nacional de Extensão são estes:

\section{Comunicação}

Cultura, Cidadania e Esporte

Meio Ambiente

\section{Educação e Formação Continuada}

Direitos Humanos e Justiça

Tecnologia e Produção

Trabalho

Saúde

Foi feito um mapeamento dos estudantes do campus estudado que desenvolveram e/ou estavam desenvolvendo atividades de Projeto de Extensão cadastrados, no período da pesquisa. Para a coleta desses dados foram utilizados os resultados de editais, encontrados no site da Instituição. Foram feitas as análises dos resultados dos últimos cinco editais. 
Primeiramente foi realizada uma verificação da quantidade de bolsas que foram ofertadas durante os cinco anos estudados

Tabela 1: Quantidade de bolsas de Extensão ofertadas para os cursos superiores

\begin{tabular}{ll} 
Ano & Quantidade de bolsas ofertadas \\
\hline $\mathbf{2 0 1 5}$ & 88 \\
$\mathbf{2 0 1 6}$ & 96 \\
$\mathbf{2 0 1 7}$ & 108 \\
$\mathbf{2 0 1 8}$ & 128 \\
\hline
\end{tabular}

Fonte: Própria(2019).

É possível observar que durante a passagem dos anos a quantidade de bolsas ofertadas para os cursos superiores foi crescente, esse fato sinaliza a preocupação da Instituição com a condição e desenvolvimento dos projetos de Extensão. Além do apoio com as bolsas ofertadas também há um apoio institucional com o cadastramento e divulgação da atividade e apoio logístico com a disponibilização de salas, materiais, transporte e outros espaços que pudessem ser utilizados para o desenvolvimento dessas atividades.

No segundo momento avaliou-se os resultados desses editais, com a verificação da quantidade de projetos aprovados pelo campus e que foram desenvolvidos em seu ano de aprovação. Pode-se explicar que a quantidade de projetos aprovados pelo curso de Bacharelado em Agronomia quando comparado com o de Licenciatura em Química, é bem maior, visto que as atividades do curso de Agronomia são de maioria práticas e precisam se desenvolvidas em campo e também pelo curso ser no período matutino e vespertino, facilitando a atividade de Extensão, quando o curso de Licenciatura ocorre apenas no período da noite, dificultando a ida á escolas e comunidades nesse período, precisando de um outro horário para tais atividades serem desenvolvidas

Tabela 2: Projetos de Extensão cadastrados na Instituição

Ano Bacharelado em Licenciatura em Química Agronomia 


\begin{tabular}{lll}
\hline $\mathbf{2 0 1 6}$ & 21 & 4 \\
$\mathbf{2 0 1 7}$ & 17 & 4 \\
$\mathbf{2 0 1 8}$ & 15 & 4 \\
\hline
\end{tabular}

Seguindo a construção dos resultados, em um terceiro momento classificamos os Planos de Trabalho cadastrados que tenham em sua construção interfaces interinstitucionais no curso de Licenciatura em Química.

Tabela 3: Projetos de Extensão cadastrados no IFPE- Campus Vitória de Santo Antão que tenham interfaces interinstitucionais

Ano Licenciatura

Em Química

\begin{tabular}{ll}
\hline $\mathbf{2 0 1 5}$ & 8 \\
$\mathbf{2 0 1 6}$ & 4 \\
$\mathbf{2 0 1 7}$ & 4 \\
$\mathbf{2 0 1 8}$ & 2 \\
\hline
\end{tabular}

Fonte: Própria (2019).

Esses trabalhos em seus objetivos continham características de atividades interinstitucionais, havendo a presença de redes de cooperação, ente o estudante extensionista e o estudante do ensino médio que em muitas vezes tem dificuldade de aprendizado em disciplinas como a de Química. A maioria dos projetos cadastrados é pertencente ao Programa Despertando Vocações Para as Licenciaturas- PDVL, este criado no ano de 2015 com o intuito de despertar vocações para a área de formação de professores, visto que é um segmento acadêmico não muito valorizado mas importante.

Alguns objetivos que estão presentes nos Planos de Trabalhos estudados são:

- Realizar atividades de experimentação em Química, numa abordagem investigativa, com vistas a auxiliar na compreensão dos conceitos e no despertar para a carreira docente na área;

-Produzir material de divulgação acerca dos aspectos relativos à licenciatura e carreira docente;

-Elaboração de materiais e atividades lúdicas a partir de jogos didáticos para o ensino de Química; 


\section{-Acompanhar os estudantes durante a inscrição do vestibular, da solicitação de dispensa de inscrição, realização de matrícula para os aprovados e durante as primeiras semanas do curso;}

-Desenvolver ações nas escolas da Rede Pública que promovam o interesse pela docência em Química através de atividades de experimentação com materiais alternativos;

Como se podem observar os objetivos desses planos de trabalho são sempre com características de auxiliar os estudantes do Ensino Médio, com uma parceria entre o orientador, orientando e a Instituição de Ensino Superior conjunto a escola que está sendo desenvolvido o projeto.

\section{Conclusões}

Por fim, este trabalho mostra a importância da extensão dentro dos cursos de graduação, para o crescimento e aprimoramento do conhecimento para esses estudantes, visto que é necessário que o aprendizado vá além da academia e que tome parâmetros de campo, respondendo problemas da sociedade por meio de extensão acadêmica. As redes de cooperação formadas pelo campus, por meio de orientador e orientando, junto á escolas da comunidade são muito importantes para a educação continuada, principalmente na condição do curso de Licenciatura, onde os estudantes ao participarem de projetos de extensão começam a lidar com o dia-dia de um professor e se prepara para sua jornada profissional e consegue ver os problemas da educação atual e busca resoluções por meio de jogos didáticos, por exemplo, facilitando o aprendizado desse estudante.

\section{Referências}

Balestrin, A., \& Verschoore, J. (2008). Redes de cooperação empresarial: estratégias de gestão na nova economia. Porto Alegre: Bookman.

BALESTRIN, A.; VARGAS,L.M.; FAYARD, P. Criação de Conhecimento nas Redes de Cooperação Inteorganizacional. Revista de Administração de Empresas, v.45, n.3, 2005.

BRASIL. Ministério da Educação. Plano Nacional de Extensão. Ministério de Educação e Cultura. Plano Nacional de Extensão, Brasília, DF, 2001.

CAGLIO, A. Networks and information technology: Competing through extranets. In: III CEMS - COMMUNITY OF EUROPEAN MANAGEMENT SCHOOLS. Louvain-la-Neuve, 7/9 maio/ 1998.

DI MAGGIO, P. J. \& POWELL,W. "the iron cage revisited" institucional isomorphism and collective rationality in organizational fields. American Sociologycal Review. 48. 14760.1983 
ECCLES, R. J. The quasi firm in the construction industry. Journal of Economic Behavior and Organizations, v. 2, n. 3, p. 335-357, 1981.

Grandori, A., \& Soda, G. (1995). Inter-firm networks: antecedents, mechanisms and forms. Organization Studies, 16(2), 183-214.

LIMA J.P.C; ANTUNES, M. T. P. A.; NETO, O.R.M.; PELEIAS, I. R. Estudo de caso e sua aplicação: proposta de um esquema teórico para pesquisas no campo da contabilidade, Ribeirão Preto, SP, v. 6, n. 14, p. 143-144, jan-abr 2012

Nohria, N., \& Eccles, R. G. (1992). Networks and organizations: structure, form, and action. Cambridge: Harvard University Press.

NONAKA, I.; TOYAMA, R.; KONNO, N. SECI, ba and leadership: a unified model of dynamic knowledge creation. In: LITTLE, S.; QUINTAS, P.; RAY, T. (Eds.). Managing knowledge an essential reader. London: Sage Publications, 2002.

NONAKA, Ikujiro e TAKEUCHI, Hirotaka. Criação do Conhecimento na Empresa: como as empresas geram a dinâmica da inovação. Rio de Janeiro: Campus,1997.

OLAVE,M.E.L., NETO, J.A. Redes de Cooperação Produtiva: Uma estratégia de competitividade e sobrevivência para pequenas e médias empresas. Gestão e Produção, v.8, n.3, p.289-303, dez, 2001.

PERROW, C. Small-Firm Networks. In: NOHRIA, N.; ECCLES, R. Networks and organizations:structure, form and action. Boston: Harvard Business School Press, 1992.

PERUCCI, R.; POTTER, H. R. Networks of power. Berlin: de Gruyter, 1989.

PROBST, G. J. B ; BÜCHEL, B. S. Organizational Learning. UK: Prentice Hall, 1997. SENGE, P. A Quinta Disciplina. São Paulo: Best Seller, 2000.

SILVA, O. da. O que é Extensão universitária. Integração: ensino, pesquisa e Extensão, São Paulo, v. 3, n. 9, p. 148- 9, maio 1997.

SILVA,K.S., MOURA, E.J., SILVA, E.V., SILVA, M.C., SILVA, E.R. Atividades de Extensão e internacionalização no curso de Agronomia no IFPE. Instituto IDV, p.8-16, 2017.

Todeva, E. (2006). Business networks. Strategy and structure. London: Routledge.

TOLBERT, P. S.; ZUCKER, L. G. A institucionalização da teoria institucional in: CLEGG,S.T.;HARDY,C.\&NORD,R.N.( org ) Handbook de estudos organizacionais. São Paulo: Ed. Atlas, 1998.

VIANA, K. S. L. Avaliação da Experiência: uma perspectiva de Avaliação para o ensino das Ciências da Natureza. 2014. 202f. Tese (Ensino de Ciências - Física e Química) Universidade Federal Rural de Pernambuco - UFRPE, Recife, 2014.

YOGUEL, G. \& KANTIS, H.: Reestructuración Industrial y Eslabonamientos Productivos: El Rol de las pequeñas y Medianas Firmas Subcontratistas. Buenos Aires, CEPAL, 1990. 